Revue d'histoire de l'Amérique française

Q4. REVUE D'HISTOIRE DE L'AMÉRIQUE FRANÇAISE

\title{
Succession et cycle familial dans le comté de Verchères, 1870-1950
}

\section{Diane Gervais}

Volume 50, numéro 1, été 1996

URI : https://id.erudit.org/iderudit/305489ar

DOI : https://doi.org/10.7202/305489ar

Aller au sommaire du numéro

\section{Éditeur(s)}

Institut d'histoire de l'Amérique française

\section{ISSN}

0035-2357 (imprimé)

1492-1383 (numérique)

Découvrir la revue

\section{Citer cet article}

Gervais, D. (1996). Succession et cycle familial dans le comté de Verchères, 1870-1950. Revue d'histoire de l'Amérique française, 50(1), 69-94. https://doi.org/10.7202/305489ar

\section{Résumé de l'article}

L'article s'intéresse à l'évolution des pratiques coutumières de la transmission du patrimoine dans le comté de Verchères entre 1870 et 1950. L'on remarque deux modèles familiaux coexistants dont l'esprit est tout à fait différent. Dans le premier, le père transmet son bien le plus tard possible, après son décès. Dans le second, le père cède son bien de son vivant, vers le moment où il souhaite prendre sa retraite. Ce dernier modèle va gagner sur l'autre. L'évolution tend vers un cycle familial qui se raccourcit, allant en quelque sorte vers l'exclusion des parents, puisque le successeur exigera d'eux qu'ils partent pour le village quand il prendra femme et achètera l'exploitation, alors que la cohabitation était autrefois d'usage. Le changement produira des tensions familiales importantes et des mécanismes facilitant la transition entre anciens et nouveaux usages se mettront en place, comme le montreront les données ethnographiques.
Tous droits réservés @ Institut d'histoire de l'Amérique française, 1996
Ce document est protégé par la loi sur le droit d'auteur. L'utilisation des services d'Érudit (y compris la reproduction) est assujettie à sa politique d'utilisation que vous pouvez consulter en ligne.

https://apropos.erudit.org/fr/usagers/politique-dutilisation/ 


\section{SUCCESSION ET CYCLE FAMILIAL DANS LE COMTÉ DE VERCHÈRES 1870-1950'}

DIANE GERVAIS IREP

Université du Québec à Chicoutimi

\section{RÉSUMÉ}

L'article s'intéresse à l'évolution des pratiques coutumières de la transmission du patrimoine dans le comté de Verchères entre 1870 et 1950 . L'on remarque deux modèles familiaux coexistants dont l'esprit est tout à fait différent. Dans le premier, le père transmet son bien le plus tard possible, après son décès. Dans le second, le père cède son bien de son vivant, vers le moment où il souhaite prendre sa retraite. Ce dernier modèle va gagner sur l'autre. L'évolution tend vers un cycle familial qui se raccourcit, allant en quelque sorte vers l'exclusion des parents, puisque le successeur exigera d'eux qu'ils partent pour le village quand il prendra femme et achètera l'exploitation, alors que la cohabitation était autrefois d'usage. Le changement produira des tensions familiales importantes et des mécanismes facilitant la transition entre anciens et nouveaux usages se mettront en place, comme le montreront les données ethnographiques.

\section{ABSTRACT}

The article studies the evolution of inheritance transmission practices in Verchères from 1870 to 1950. Two models of completely different spirit co-existed in this rural county. In the first, only death could separate the father from his property; in the second, the father passed on the farm while he was still alive, at the time of retirement. Over the period studied, the second model gained in currency at the expense of the first. This change favoured a shorter family life-cycle. In a sense, it also represented the exclusion of the older generation, since upon marrying and purchasing the farm, the heir demanded that the parents depart for the village rather

1. Le projet «Saint-Laurent» bénéficie d'une subvention accordée à Gérard Bouchard par la Fondation de l'Université du Québec à Chicoutimi. L'auteure, pour sa part, a reçu l'appui financier du Conseil de recherches en sciences humaines du Canada (CRSH) et du Fonds FCAR, qu'elle remercie. Elle remercie aussi les précieuses collaboratrices qu'ont été Geneviève Postolec, pour le dépouillement des archives de l'Enregistrement, et Martine Hamel pour le dépouillement des données de recensement. Elle est également reconnaissante envers Gérard Bouchard, Yves Otis, Gérard Béaur, Bernard Derouet et Antoinette Fauve-Chamoux pour leurs excellentes suggestions, ainsi qu'envers Joseph Goy, pour son appui indéfectible.

RHAF, vol. 50, nº 1, été 1996 
than remain and co-habit as had previously been the norm. While it generated considerable tensions within the family, the change also provoked the creation of mecanisms to facilitate the transition from the old system to the new one. This ethnographic data confirm.

\section{LE PROJET SAINT-LAURENT}

Dans un article publié il y a quelques années dans les Annales, Gérard Bouchard exposait la problématique d'un projet de comparaison des modèles de la reproduction familiale observés en France et au Québec. L'hypothèse de départ repose sur la distinction entre les systèmes de reproduction ouverts des sociétés neuves, à tendance égalitaire, comme le Saguenay par exemple, et les systèmes clos, à l'exemple des vieux pays européens, que sont devenus les vieux terroirs laurentiens, une fois toutes les terres occupées, défrichées et mises en valeur. La saturation du terroir s'accompagnerait de modifications significatives des pratiques de transmission et d'établissement des enfants des familles d'agriculteurs. Devant la rareté des terres, le père le mieux intentionné, se voit dans l'impossibilité d'établir tous ses enfants, au risque de l'inégalité. De ce point de vue, la reproduction familiale est entendue comme un système de stratégies et d'aménagements "propres à restaurer l'équilibre entre le nombre de candidats à la terre et l'espace disponible». Les recours possibles sont nombreux. Grosso modo, Bouchard les répartit entre quatre grandes options: agir sur la démographie, exclure des enfants de l'héritage ou de la succession, réaliser d'une manière ou d'une autre l'expansion du patrimoine nécessaire à tous les établissements et, enfin, mettre en œuvre des dispositions à caractère social, telles que l'indivision ou la cohabitation. Bouchard exhorte enfin l'historien ou l'ethnologue qui s'y penchera, à reconnaître l'ampleur et la nature des inégalités entraînées par les pratiques familiales ainsi que les ruptures et les changements résultant de tensions mal résorbées ou de contradictions insurmontables, de même qu'à identifier les sources de la diversité des stratégies inscrites dans les traditions locales ${ }^{2}$.

C'est dans ce cadre que s'inscrit le «Projet Saint-Laurent», initié à l'Institut interuniversitaire de recherches sur les populations (IREP), qui vise plus modestement la comparaison de trois vieux terroirs laurentiens, soit l'île d'Orléans, le comté de Champlain et celui de

2. Gérard Bouchard, «La reproduction familiale en terroirs neufs. Comparaison sur des données québécoises et françaises», Annales ESC, 2 (mars-avril 1993): 424. Pour le projet de comparaison des vieux terroirs québécois, voir Gérard Bouchard et Diane Gervais, Le projet StLaurent. Problématique et grille d'entrevue, Doc. IREP, II-C-231, 1993. 
Verchères. Cet article rend compte d'un premier terrain d'enquête, celui de Verchères, et se penche précisément sur l'évolution d'un système de pratiques coutumières de la transmission des exploitations familiales entre les années 1870 et 1950.

\section{PRATIQUES COUTUMIÈRES DE LA SUCCESSION À VERCHÈRES}

Trois paroisses du comté de Verchères ont déjà fait l'objet de recherches historiques s'intéressant à la reproduction familiale ${ }^{3}$. Louis Michel s'est penché sur les paroisses de Varennes et de Verchères pour la période qui va des origines jusqu'en 1850 . Son étude s'arrête vers le point de saturation des terres, visible dans le plafonnement des possibilités d'établissements agricoles. Durant la période considérée, Louis Michel note la grande variété des pratiques notariales utilisées par les agriculteurs dans la transmission de leur patrimoine. La majorité de ces pratiques sera toujours d'usage dans le dernier tiers du XIX ${ }^{\mathrm{c}}$ siècle, mais la plupart auront disparu en 1950 où la nouvelle pratique de transmission par la vente du père à son fils successeur aura uniformisé les usages.

Dominique Joulia a aussi étudié la paroisse de Varennes, mais pour les premières décennies du $\mathrm{XIX}^{\mathrm{e}}$ siècle seulement. Dès cette époque, Joulia perçoit l'émergence de clivages sociaux dans cette société agricole jusqu'alors relativement homogène. Une hiérarchisation des enfants à l'intérieur des familles, de même qu'entre les lignées et entre les familles est dessinée par des stratégies d'alliances privilégiées entre certaines familles et au gré des transmissions foncières inégalement réparties.

Pour sa part, Pauline Desjardins a étudié les chaînes de titres et les archives de quinze exploitations de l'ancienne paroisse de SainteThéodosie depuis 1730 jusqu'en 1975. Cette dernière monographie possède, entre autres mérites, celui de précéder, de chevaucher et de dépasser la période de saturation des terres agricoles jugée critique pour les possibilités d'établissement des nombreux enfants des familles d'agriculteurs, ce qui permet d'observer, en effet, un modèle

3. Louis Michel, «Varennes et Verchères des origines au milieu du XIX $X^{\mathfrak{e}}$ siècle. État d'une enquête», dans Évolution et éclatement du monde rural. France-Québec XVII -XXe siècles (Paris/Montréal, 1986), 325-340. Pauline Desjardins, «La Coutume de Paris et la transmission des terres. Le rang de la Beauce à Calixa-Lavallée de 1730 à 1975», Revue d'histoire de l'Amérique française, 34,3 (décembre 1980): 331-339. Dominique Joulia, «Pratiques successorales en milieu rural 1795-1870. Étude comparative de cas. France de l'Ouest-Québec», dans Étude comparative de la société rurale de la France de l'Ouest et du Québec (XVII -XX siècles) (Montréal/Paris, Université de Montréal/École des hautes études en sciences sociales, 1981), 93-141. 
de reproduction familiale en porte-à-faux avec l'égalitarisme de principe de l'époque pionnière.

Le comté de Verchères apparait à première vue bien étudié. Pourtant, sauf pour la paroisse de Calixa-Lavallée, la période contemporaine demeure inexplorée. En outre, les trois études précitées sont fondées sur des sources historiques exclusivement. Au contraire, cet article ajoute au précieux corpus d'archives notariales un important matériel ethnographique fondé sur des témoignages oraux. D'autre part, pour suivre l'évolution des pratiques familiales et sociales dans la longue durée, il fallait privilégier un terrain de recherche où les périodes antérieures avaient été bien documentées. La comparaison dans le temps bénéficie ainsi d'un appui solide, grâce à ce passé mis au jour.

Le contexte de la recherche étant posé, précisons la notion de "pratiques coutumières», telle qu'utilisée ici. Les pratiques coutumières renvoient à des usages partagés par l'ensemble ou une partie d'une communauté, dont on peut observer la récurrence ou la régularité statistique sur une période donnée. Suivant Pierre Bourdieu, la régularité de telles pratiques provient de ce qu'elles sont intériorisées par les membres de la collectivité, un peu comme si ces derniers possédaient une sorte de «sens du jeu» qui fait qu'ils se comportent d'une certaine manière en certaines circonstances ${ }^{4}$.

La permanence et la cohérence d'un système de pratiques coutumières demeurent fragiles toutefois, car ces manières de faire répétées au fil des générations s'expriment dans des contextes sociaux et idéologiques changeants. Acquises et transmises par l'observation plus ou moins consciente des manières de faire des voisins, des parents et des anciens, les pratiques coutumières sont sans cesse réexaminées par les générations successives et n'échappent ni à la critique ni aux manipulations. Communément partagées à un moment donné de l'histoire d'une collectivité, elles peuvent être plus tard remises en question, comme on le verra à l'analyse de notre terroir laurentien.

Rappelons enfin les quelques principes de l'organisation des fermes familiales qui sont à la base de l'argumentation. En milieu agricole, il faut voir les relations familiales et les relations de travail dans une structure unitaire. L'objet de la transmission (terres, maisons, bâtiments, animaux et roulant de ferme) constitue à la fois le cadre de vie et les moyens de production d'une famille. Plus qu'un simple

4. Pierre Bourdieu, «De la règle aux stratégies», Choses dites (Paris, Éditions de Minuit, 1987), 75-94. 
transfert de bien, la transmission d'un bien d'exploitation agricole opère de surcroît un transfert des responsabilités, du droit au travail, aux prises de décisions et à la perception des revenus. Bref, la transmission d'un bien agricole est indissociable de la succession.

Par ailleurs, la succession ne s'avère pas une relation à sens unique des parents vers le fils, mais plutôt une relation bilatérale entre deux parties aux intérêts divergents: l'une qui souhaite prendre la place et l'autre qui ne souhaite peut-être pas la céder trop vite. À l'égard du retrait des parents, plusieurs scénarios sont envisageables. En effet, les parents peuvent se retirer d'une manière ponctuelle et définitive ou, au contraire, céder leurs biens et prérogatives de façon progressive, suivant un processus qui s'étale dans le temps. Ainsi en est-il par exemple des pratiques où le père ne cède dans un premier temps que la nue-propriété de l'exploitation, en se réservant un droit d'habitation dans la maison située sur le bien, ainsi que le droit de percevoir les revenus jusqu'à son décès. Il en va de même du moment de la retraite du père dans le cycle familial (ou cycle parental). Le père peut se retirer très tôt ou, au contraire, très tardivement, ce qui n'est pas sans conséquence pour le fils successeur.

Pour le père comme pour le fils, le calendrier de la succession, les conditions et les modalités particulières de la transmission des biens et des pouvoirs sont déterminants pour le cours de leur destin: agissant sur la durée de la cohabitation, ils modulent la structure des ménages qui se remplacent sur le bien, tout comme la qualité des relations intrafamiliales; même le choix du successeur y apparaît subordonné.

Les pratiques coutumières de la reproduction familiale débordent largement la simple question de la succession. Pour assurer la continuité des exploitations familiales, les agriculteurs doivent planifier leur succession et transmettre leur bien tout en assurant leur propre subsistance jusqu'à leur décès. Il leur faut en outre régler la question de l'héritage et du destin de leurs nombreux enfants dans un esprit d'équité. Cette dernière question ne sera pas abordée ici; seule l'analyse du processus de succession et de transmission de l'exploitation agricole sera traitée. Il ne sera question que de la relation des parents à leur fils successeur, mettant en veilleuse le problème de l'exclusion des autres enfants. L'objectif est de mettre en relief une modification très significative du cycle familial, entraînée au $X^{e}$ siècle, par le recul de la pratique de la cohabitation des parents donateurs avec leur successeur et sa famille.

L'article traitera en premier lieu des usages relatifs au choix du fils successeur et à son rang dans la fratrie. L'on s'intéressera ensuite à la structure des ménages agricoles, puis au calendrier de la 
succession et à ses effets sur la qualité des relations familiales. Considérant l'importance des changements survenus au cours de la période, nous verrons ensuite les mécanismes régulateurs de la transition et évoquerons, enfin, les effets néfastes de la mésadaptation aux nouveaux usages pour la continuité des exploitations familiales.

\section{SOURCES ET MÉTHODE}

Dans la construction d'un modèle structurant des pratiques et des représentations, l'apport de l'ethnographie aux sources historiques est inestimable, puisqu'elle permet d'intégrer à l'analyse le discours des acteurs eux-mêmes. L'étude s'appuie donc sur des témoignages oraux et sur des sources d'archives: essentiellement les archives notariales du Bureau d'enregistrement d'une paroisse du comté ${ }^{5}$. Plus accessoirement, les données des recensements canadiens situent dans un cadre plus général les stratégies familiales dont on veut rendre compte.

Selon la nature de la source, des «dossiers de famille» et des «dossiers d'exploitation» ont été constitués. Chaque dossier couvre l'ensemble de la période. Les dossiers d'exploitation sont fondés sur les actes de l'enregistrement. Leur fil conducteur, c'est le sol: l'ensemble des lots qui constituent une exploitation. Les dossiers fondés sur l'enregistrement renseignent sur la succession des mutations foncières ainsi que sur les dettes et les hypothèques affectant chaque lot. Ils rendent compte des modalités des transmissions intergénérationnelles, comme des ventes à des étrangers à la famille, permettant dans une certaine mesure d'évaluer la mobilité des familles.

Les dossiers de famille, quant à eux, sont construits à partir d'entretiens menés auprès de fils et de filles d'agriculteurs. Leur fil conducteur est la généalogie des familles. Un minimum de 9 heures d'entretien a été exigé de chaque témoin. Certains, plus disponibles, ont été beaucoup plus loin. La longueur des entretiens est due à l'importance de la grille d'enquête, de même qu'à un mode d'entretien

5. Le dépouillement des actes notariés du Bureau de l'enregistrement de Sainte-Julie a été fait par sondage, dans une seule paroisse du comté, en retenant un lot sur vingt. Les dossiers d'exploitations complètes sont constitués par l'addition des lots repérés grâce au dépouillement des actes du premier lot sélectionné. La période couverte par l'enregistrement va de 1878 à nos jours. Les généalogies des propriétaires et des «parties» mentionnées dans les actes ont été reconstituées aux Archives nationales du Québec. Pour les enquêtes orales, un principe de diversité des informateurs a été appliqué: diversité quant au sexe, à la profession et au statut matrimonial. Pour protéger l'anonymat des sources, ni les noms des personnes ni les paroisses où nous avons travaillé ne sont divulgués. Des pseudonymes seront utilisés au besoin. Pour la méthodologie du projet, voir Diane Gervais, Le projet St-Laurent. Guide de collecte des données. Guide pratique à l'intention des assistants de recherche, Doc. IREP, II-C-227, 1994, 46 p. 
semi-directif, où la trame du destin de chaque individu a été progressivement extraite de la mémoire et patiemment reconstituée. La grille d'enquête doit beaucoup à Gérard Bouchard. Elle couvre l'ensemble des paramètres de la reproduction familiale: histoire et nature de l'exploitation, généalogie de trois générations, destins des individus, modalités des transferts intergénérationnels et, enfin, représentations familiales.

L'aire de recherche dépasse les limites de la paroisse. Le hasard des rencontres, dans la recherche des témoins, a entraîné un recrutement dans cinq paroisses du comté de Verchères. L'homogénéité régionale pressentie, au vu de la fréquence des intermariages entre les paroisses, ne s'est pas démentie. La démonstration s'appuie sur l'histoire de 35 exploitations agricoles, comparant les biographies de près de 600 individus regroupés en 75 fratries. Une récurrence satisfaisante des comportements types dégagés permet d'estimer les tendances locales des pratiques coutumières de la succession, pour la période considérée.

\section{UNE PRATIQUE STABLE: LE CHOIX DU SUCCESSEUR}

Parmi les pratiques coutumières de la transmission familiale, considérons en premier lieu le choix du successeur. Glissons d'abord un mot sur le cadre familial des successions. Dans le comté, les familles d'agriculteurs sont nombreuses: l'on compte facilement $10 \mathrm{ou}$ 11 naissances par famille. Les grandes familles de 15 à 20 enfants, quoique beaucoup plus rares, suscitent l'admiration et sont citées en exemple. La pensée générale est qu'il n'y a rien de plus beau que les enfants. «Les enfants, ça n'appauvrit pas», dit-on, pour signifier qu'ils sont une richesse.

Dans les 75 familles retenues, on peut encore retracer près de 8 enfants en moyenne par famille, au moment où se pose la question de la succession. Qui donc succédera à son père dans cette fratrie relativement importante? Pas de surprise, ce sera un garçon et il devra se marier. En effet, malgré une assez forte incidence du célibat masculin (12\% au recensement de 1901), on ne retrouve pas de célibataires dans le rang des successeurs, sauf dans les dernières décennies de la période étudiée 6 .

L'âge au mariage du fils successeur est très variable: entre 21 ans et 59 ans, pour les 33 successeurs dont l'âge au mariage est connu.

6. Le nombre d'enfants a été obtenu après avoir éliminé les enfants au destin inconnu ainsi que les enfants décédés avant d'avoir atteint l'âge adulte. Pour la proportion des célibataires, voir les manuscrits du recensement canadien de 1901, pour cinq paroisses du comté de Verchères. 
L'âge au mariage du successeur apparaît très dépendant de la «donne» familiale. Il agit, de fait, comme régulateur. Le décès prématuré de la mère ou du père, ou le mauvais état de santé de l'un ou de l'autre, aura pour effet de précipiter le mariage du successeur et, éventuellement, la transmission; mais là où les parents manifestent une ferme volonté de préserver leurs droits sur le bien d'exploitation, le mariage du successeur pourra être retardé indûment.

Par contre, le rang du successeur dans la fratrie est assez constant. Il est en effet remarquablement fréquent de retrouver le successeur parmi les derniers fils à se marier. Dans les deux tiers des cas, le successeur est choisi dans la deuxième moitié de la fratrie. La tendance est nette et confirme les dires des témoins interrogés quant à la logique du choix du successeur: celui qui aura le bien est généralement le fils qui se mariera vers le moment où le père souhaite prendre sa retraite. La pratique est attestée dans d'autres régions du Québec, notamment par des études à caractère ethnologique ${ }^{7}$.

En réalité, au moment où se pose le problème de la reprise, vers l'âge de la retraite du père, la plupart des enfants ont déjà quitté la maison familiale et sont établis dans la vie. C'est ainsi que le vieux bien familial est naturellement transmis à l'un des derniers fils et que ce choix peut emporter consensus. Une topique très présente dans le discours des témoins veut que le destin des enfants, garçons ou filles, soit prédéterminé par leur rang dans la famille, comme par une sorte de fatalité. Plusieurs cherchent à excuser des parents qui, disent-ils, ont fait de leur mieux ${ }^{8}$. Un témoin dit par exemple: «J'aurais aimé être agriculteur, mais j'étais l'aîné de 12 enfants. J'ai dû aller travailler jeune. Je donnais mon salaire aux parents: $\mathrm{j}$ 'ai pas pu ramasser de quoi acheter une terre.»

La stratégie coutumière qui consiste, pour les parents, à désigner comme successeur l'un de leurs derniers fils favorise un cycle familial long. Un cycle long aura pour effet de réduire de façon significative la durée de la phase de cohabitation qui semble d'usage entre les

7. Voir les études de C.-H.-P. Gauldrée-Boileau, «Paysan de Saint-Irénée de Charlevoix en 1861 et 1862», dans Paysans et ouvriers québécois d'autrefois, Les Cahiers d'Institut d'histoire (Québec, 1968), 11: 59; Marcel Rioux, «Description de la culture de l'île Verte», 133: 32; Horace Miner, St. Denis. A French-Canadian Parish (Chicago, 1939), 82; Léon Gérin, «L'habitant de St-Justin», dans Léon Gérin et l'habitant de St-Justin (Montréal, 1968), 88.

8. La citation est tirée du dossier de famille $\mathrm{n}^{\circ} 33$; voir l'annexe relative aux dossiers cités. Gérard Bouchard estime que le choix du successeur n'est pas déterminé par le rang de naissance, mais par l'âge du père au moment où il décide de se départir de son bien: «Rang de naissance et destin socio-économique dans la région du Saguenay (XIX ${ }^{e}-X^{e}$ siècles)», dans M. Segalen, G. Ravis-Giordani, dir., Les cadets (Paris, 1994), 100. Les deux variables apparaissent difficiles à dissocier. 
parents et le jeune ménage qui prend le relais sur le bien d'exploitation. L'analyse de la structure des ménages, d'après les données des recensements de 1891 et de 1901, permettra d'explorer cette hypothèse.

\section{UNE STRATÉGIE D'ÉVITEMENT DE LA COHABITATION?}

La grille de classification des ménages, telle que conçue par Peter Laslett, a été légèrement modifiée par Gérard Bouchard et Jeannette Larouche ${ }^{9}$. C'est cette dernière grille qui a été appliquée aux données des recensements nominatifs de cinq paroisses du comté de Verchères, en lui apportant toutefois une modification supplémentaire. Pour apprécier la durée de la cohabitation entre les parents donateurs et le jeune couple qui succède, nous avons adapté la méthode suggérée par Lutz Berkner dans son analyse des ménages d'une région de la BasseAutriche au XVIII ${ }^{\mathrm{e}}$ siècle.

Sans vouloir relancer un débat déjà bien documenté, il est utile de rappeler, comme le fait Berkner, que la terminologie de la classification des ménages ne correspond pas véritablement à une typologie des ménages. Il faut chercher à corriger l'image statique des organisations familiales livrée par l'analyse des recensements et considérer l'évolution cyclique des ménages dans le temps. En effet, la composition des ménages à structure complexe est soumise à de grandes variations dans le temps; c'est ce qu'on appelle le cycle familial. À la veille du mariage de leur successeur, le ménage composé des parents et de leurs enfants est dit «simple» puisqu'il ne comprend qu'un seul noyau conjugal. Lorsque le successeur se marie, jusqu'au décès du premier mourant des parents, le même ménage est classé «multifamilial», c'est-à-dire dans la catégorie des ménages composés de deux noyaux conjugaux apparentés. Après le décès d'un parent, le ménage sera classé dans les «ménages à famille élargie», formés d'un couple, de leurs enfants et d'un parent veuf. Après le décès du parent veuf, le ménage redevient $«$ simple $^{10} »$.

9. Gérard Bouchard et Jeannette Larouche, Enquête sur la structure des ménages saguenayens. Présentation de la typologie, Doc. IREP, II-C-187, juin 1991, 11 p. Les problèmes de méthode de l'analyse des recensements, soulevés notamment par Gilles Lauzon, n'ont pas été pris en compte. Les maisons à logements multiples sont beaucoup moins répandues à la campagne qu'à la ville. Quelques-unes existent dans les villages du comté et il se trouve quelques rares maisons dites «à deux côtés» dans les rangs. Une petite proportion des ménages identifiés comme multifamiliaux risque peut-être de recouvrir une cohabitation dans des logements séparés. G. Lauzon, «Cohabitation et déménagements en milieu ouvrier montréalais. Essai de réinterprétation à partir du cas du village de Saint-Augustin (1871-1881)», RHAF, 46,1 (été 1992): 115-142.

10. Lutz K. Berkner, «The Stem Family and the Developmental Cycle of the Peasant Household: an Eighteen-Century Austrian Exemple», The American Historical Review (1972): 
Pour l'essentiel, Berkner démontre que la méthode habituelle de calcul de la fréquence des ménages multifamiliaux ne permet pas d'apprécier à sa juste valeur la dominance de ce modèle d'organisation familiale dans une communauté. Étant donné que la mort des parents brisera un jour ou l'autre le double ménage, il suggère de ne prendre en compte que les jeunes ménages et non pas l'ensemble des ménages.

La grille a donc été appliquée aux ménages de Verchères dont le chef de famille (ou l'un des chefs de famille, dans les cas de ménages multifamiliaux) est âgé de moins de 35 ans (voir le tableau 1). Comparés à l'ensemble des ménages, ces jeunes ménages montrent une proportion plus importante de ménages à deux noyaux apparentés. En

TABLEAU 1

Structure des ménages dans cinq paroisses du comté de Verchères

\begin{tabular}{|c|c|c|c|c|}
\hline & \multicolumn{2}{|c|}{ Tous les ménages } & \multicolumn{2}{|c|}{ Jemnes ménages } \\
\hline & 1891 & 1901 & 1891 & 1901 \\
\hline 1. Ménages sans noyau familial & $\begin{array}{r}5,9 \% \\
(67)\end{array}$ & $\begin{array}{l}9,7 \% \\
\text { (112) }\end{array}$ & $\begin{array}{r}0,0 \% \\
(0)\end{array}$ & $\begin{array}{r}3,1 \% \\
(9)\end{array}$ \\
\hline 2. Ménages simples & $\begin{array}{r}74,1 \% \\
(829)\end{array}$ & $\begin{array}{r}72,8 \% \\
(8.38)\end{array}$ & $\begin{array}{r}68,4 \% \\
(2(17))\end{array}$ & $\begin{array}{r}70,6 \% \\
(204)\end{array}$ \\
\hline 3. Ménages a famille élargie & $\begin{array}{r}10,3 \% \\
(115)\end{array}$ & $\begin{array}{r}13,2 \% \\
(152)\end{array}$ & $\begin{array}{r}6,2 \% \\
(19)\end{array}$ & $\begin{array}{r}11,1 \% \\
(32)\end{array}$ \\
\hline \multicolumn{5}{|l|}{ 4. Ménages imultifamiliaux } \\
\hline a) par apparentememt & $\begin{array}{r}6,0 \% \\
(68)\end{array}$ & $\begin{array}{r}4.0 \% \\
(45)\end{array}$ & $\begin{array}{r}19.1 \% \\
\quad(58)\end{array}$ & $\begin{array}{r}13,8 \% \\
(40)\end{array}$ \\
\hline h) sans apparentement & $\begin{array}{r}3,7 \% \\
(41)\end{array}$ & $\begin{array}{r}0,3 \% \\
\text { (4) }\end{array}$ & $\begin{array}{r}6,3 \% \\
\text { (19) }\end{array}$ & $\begin{array}{r}1.4 \% \\
(4)\end{array}$ \\
\hline Total & $\begin{array}{l}9,7 \% \\
(109) \\
\end{array}$ & $\begin{array}{r}4,3 \% \\
(49) \\
\end{array}$ & $\begin{array}{r}25.4 \% \\
(77) \\
\end{array}$ & $\begin{array}{r}15,2 \% \\
\quad(44) \\
\end{array}$ \\
\hline TOTAL & $\begin{array}{c}100 \% \\
(1 \quad 120)\end{array}$ & $\begin{array}{c}100 \% \\
(1151)\end{array}$ & $\begin{array}{c}100 \% \\
(30.3)\end{array}$ & $\begin{array}{l}100 \% \\
(289)\end{array}$ \\
\hline
\end{tabular}

Sources: Manuscrits des recensements canadiens de 1891 et 1901. Dépouillement: Martine Hamel (IREP).

398-418. Pour une estimation plus sûre de la cohabitation intergénérationnelle, il faut observer les ménages multifamiliaux apparentés, plutôt que les autres structures. Le cycle familial d'un ménage complexe peut comporter de longs moments de structure simple, comme la famille élargie peut recouvrir un grand nombre de situations; par exemple, au Québec, le parent veuf accueilli par un enfant marié. Voir l'analyse d'Antoinette Fauve-Chamoux, «Les structures familiales en France aux XVII ${ }^{e}$ et XVIII ${ }^{\mathrm{e}}$ siècles», dans J. Dupaquier, dir., Histoire de la population française (1988), 2: 335-336. 
1891, la proportion passe de $6 \%$ à $19,1 \%$ et en 1901 , de $4 \%$ à $13,8 \%$. La très grande majorité des ménages multifamiliaux recensés pour l'ensemble de la population se retrouve chez les jeunes ménages. En 1891, 58 des 68 ménages multifamiliaux sont de jeunes ménages, tandis qu'en 1901, l'on retrouve 40 des 45 ménages multifamiliaux parmi les jeunes ménages, soit la quasi-totalité. Un tel résultat était somme toute attendu et fait valoir la durée relativement courte de la cohabitation.

Si l'on considère la taille des familles et le nombre de jeunes ménages qui ne sont pas bénéficiaires d'une succession, mais s'installent tout de même dans la paroisse, la fréquence observée pour les ménages multifamiliaux apparaît significative de la pratique coutumière de la «famille-souche» dans la transmission des biens ruraux au tournant du siècle. Quant au caractère relativement temporaire de la cohabitation, il est le résultat de la stratégie qui favorise comme successeur l'un des derniers garçons de la fratrie. S'agit-il d'une stratégie consciente d'évitement? Voilà une question à laquelle nous ne saurions répondre pour l'instant.

\section{MODÈLES FAMILIAUX ET CALENDRIER DE LA SUCCESSION}

Les pratiques familiales reliées au calendrier de la succession mettent en évidence les tensions inhérentes au processus de transmission. Elles opèrent un clivage si net entre les familles que l'on peut $\mathrm{y}$ voir le reflet de deux modèles familiaux coexistants. Les deux modèles persistent tout au long de la période, bien que l'un d'eux régresse avec le temps.

Prenons d'abord quelques mesures de la durée des deux calendriers types. La première tendance, qui va dominer finalement, consiste pour le père à donner ou à vendre son bien vers le moment du mariage du successeur. L'âge moyen du père, au moment de la transmission, est alors de 62 ou 63 ans, assez près de ce que l'on a considéré longtemps comme l'âge normal de la retraite. La vie active du père et son pouvoir de gestion sur ses affaires durent alors en moyenne 38 ans, pour peu qu'il ait lui-même reçu son bien au mariage et qu'il cède en même temps que le bien, le droit d'en percevoir les revenus. En effet, aussi longtemps que les parents se réservent un droit d'usufruit, l'on ne peut considérer le processus de transmission complété.

L'autre tendance est illustrée de manière éloquente par un témoin. «Mon père a été propriétaire toute sa vie!... Avant de se coucher pour l'éternité, faut pas se déshabiller trop vite!» Dans ce modèle familial beaucoup plus autoritaire, la transmission est opérée par voie testa- 
mentaire. Généralement, sauf en cas de maladie grave, le père rédige très tard son testament, après l'âge de 70 ans. Mais à la suite d'une réserve d'usufruit très habituelle en faveur de la veuve, le fils ne sera propriétaire en titre qu'après le décès de ses deux parents. L'écart moyen entre la date du mariage du père et celle du transfert de propriété s'élève alors à 53 ans, soit 15 ans de plus que si le père avait donné son bien au mariage de son fils. Pour le fils, cela signifie que, longtemps après son mariage, il ne jouit pas encore des revenus de son travail (les chèques sont libellés au nom du père) et qu'il n'est pas maître des décisions importantes. Le père "garde la main haute», comme disent les témoins. La première tendance implique, pour le père, un cycle familial plus court que dans le second cas, mais la stratégie commune du choix d'un successeur vers la fin des fratries montre somme toute une disposition assez partagée en faveur d'un cycle familial long.

\section{L'ESPRIT DE LA PRATIQUE NOTARIALE}

Le calendrier de la succession oriente le choix du type de transaction notariale. L'un et l'autre sont déterminants pour le statut du successeur dans sa famille d'origine et, de façon générale, pour la qualité des relations familiales. A tous ces égards, les pratiques familiales évolueront notablement. À la fin du XIX $\mathrm{X}^{\mathrm{e}}$ siècle, les familles se partagent entre celles où l'exploitation est transmise du vivant des parents, par donation, et les familles qui ont recours à la transmission testamentaire. Au XX $X^{e}$ siècle, la donation disparaît au profit de la vente du père à son fils, tandis que recule le testament ${ }^{11}$. L'évolution de la pratique notariale emporte avec elle le recul de la cohabitation entre les parents donateurs et le ménage du successeur.

\section{Modèles familiaux de la fin du XIXe siècle}

Examinons de plus près les cultures familiales sous cet angle ${ }^{12}$. À la fin du XIX ${ }^{\mathrm{e}}$ siècle, le paysage coutumier se partage également entre les pratiques de donation de ferme et de transmission par voie testamentaire. La donation de ferme implique la cohabitation des parents

11. Pauline Desjardins note une évolution similaire de la pratique notariale des successions pour la paroisse de Calixa-Lavallée: loc. cit., 335-337.

12. Pour observer l'évolution des pratiques, les fratries ont été partagées en deux groupes. Le premier rassemble les fratries dont les parents se sont mariés avant 1893, tandis que le second regroupe les fratries dont les parents se sont mariés après cette date. L'année 1893 correspondant à la plus ancienne date de mariage des parents du plus âgé des témoins, toutes les fratries des témoins tombent dans la tranche temporelle la plus récente. Une étude longitudinale de chaque dossier, encore à faire, raffinera l'analyse des cycles familiaux. 
donateurs avec le successeur qui fait entrer sa femme dans la maison. Tous travaillent ensemble et vivent «au même pot et au même feu», selon l'expression consacrée. Au moment de la signature de l'acte notarié, le jeune ménage s'engage à prendre à sa charge ses vieux parents et ses frères et sœurs célibataires. La donation entraîne une inversion des responsabilités et des charges. Le fils donataire, devenu en droit propriétaire du bien, devient chef de famille, c'est-à-dire que ses frères et sœurs qui vivent sous son toit lui sont subordonnés. Par contre, ses rapports avec ses parents demeurent ambigus, car il est difficile pour les parents d'abdiquer leur autorité du jour au lendemain. L'ascendant somme toute bien naturel des parents sur leurs enfants ne peut se voir balayé d'une simple signature apposée sur une transaction notariale ${ }^{13}$.

Il en va autrement des familles où le père transmet son bien par testament. Dans ce cas, le fils ne devient propriétaire en titre qu'après le décès de ses parents. Les conditions de la cohabitation du successeur avec sa famille d'origine paraissent à première vue identiques. Pourtant, quelque chose fait toute la différence: c'est le statut du fils successeur dans la famille et dans son rapport au bien familial. Même marié et chef de famille, il est perçu et se perçoit lui-même comme un mineur, soumis à ses parents tant que ceux-ci vivront.

En effet, dans son testament, le père lègue son bien en partageant la propriété entre son épouse survivante et son successeur suivant des modalités qui accordent à son épouse les pouvoirs de gestion du bien. Comme le dit laconiquement un témoin: «La mère garde les revenus, mais elle ne peut pas vendre.» Pauline Desjardins note aussi cette disposition dans les testaments de la paroisse de Calixa-Lavallée à partir de la fin du XIX ${ }^{\mathrm{c}}$ siècle, par laquelle l'usufruit de l'ensemble des biens est légué à l'épouse et la nue-propriété au successeur ${ }^{14}$. Pour le fils, cela signifie qu'il ne sera propriétaire à part entière qu'après le décès de sa mère veuve. Une telle mesure évite le paiement d'un droit de mutation supplémentaire, assure la protection de la veuve en lui laissant le contrôle des revenus et permet éventuellement d'éviter la vente qui pourrait suivre un décès prématuré du père. Par contre, elle prolonge la minorité du fils successeur au-delà d'une limite qui

13. Les donations de fermes ont été beaucoup étudiées. Voir notamment les analyses très pertinentes de Santerre et Bouchard. Renaud Santerre, «Donations de fermes et sécurité des agriculteurs âgés, 1850-1990», dans G. Bouchard et J. Goy, dir., Famille, économie et société rurale en contexte d'urbanisation (17e-20e siècle) (Chicoutimi/Paris, 1990). Gérard Bouchard, Quelques arpents d'Amérique. Population, économie, famille au Saguenay 1838-1971 (Montréal, Boréal, 1996), 203-210.

14. Pauline Desjardins, loc. cit., 336. 
paraîtra de plus en plus abusive à mesure que l'on avance dans le $\mathrm{XX}^{\mathrm{e}}$ siècle.

Bref, malgré des divergences significatives quant à l'emprise des parents sur leurs enfants, les modèles d'organisation familiale de la fin du $\mathrm{XIX}^{\mathrm{e}}$ siècle se caractérisent par un cycle familial long, par une phase de cohabitation généralement brève avec les parents et par des pratiques communautaires d'exploitation du bien familial fondées sur la hiérarchisation des frères et sœurs corésidents.

\section{Modèles familiaux de la première moitié du XXe siècle}

Considérons maintenant les familles constituées au tournant du siècle. Le partage de ces familles suivant le dispositif de dévolution patrimoniale opère le même clivage entre les transmissions testamentaires et les autres. Le testament régresse toutefois. Le tiers des parents seulement persistent à utiliser ce mode autoritaire de transmission. Les conséquences en seront dramatiques pour les successeurs: soit ils se marieront tardivement, soit ils demeureront célibataires, car les femmes ne voudront plus entrer dans ces maisons où les parents s'accrochent au bien.

Pour les autres familles (soit les deux tiers des familles), les choses vont changer. La transmission du bien familial par voie de donation et les pratiques familiales communautaires qu'elle impliquait seront dépassées. La vente du bien devient la norme. La dynamique familiale en sera bouleversée, car la vente transforme radicalement les modalités de l'échange intergénérationnel. En effet, si l'on examine la transmission intergénérationnelle dans une perspective échangiste, comme un don qui appelle un retour, l'on est amené à considérer les modalités particulières du retour de don, ou des contreparties attachées à la succession, comme constituant les assises de la construction du lien familial.

Dans les actes de donation, les compensations dues par le successeur à ses frères et sœurs non encore établis, ainsi que les dispositions aménageant la cohabitation avec les parents, étaient amplement développées. Toutes ces dispositions disparaissent des actes de vente. Les sources orales confirment aussi le départ des parents après la vente: lorsque le fils marié achète le bien, les parents partent avec le reste de la famille, célibataires adultes et jeunes enfants, s'il en reste.

La monétarisation de la succession va modifier l'ancien équilibre des rapports entre les personnes. La vente implique une contrepartie en espèces sonnantes qui annule le sentiment de dette, voire peut-être le devoir de reconnaissance. Les sommes versées facilitent la sépa- 
ration des ménages du successeur et de ses parents, laquelle met un terme à l'interdépendance et aux contraintes de la vie communautaire ${ }^{15}$. En outre, et ce n'est pas rien, une fois le bien vendu, aucun membre de la famille, à l'exception du successeur, n'a de droit sur le bien, à quelque titre que ce soit. En même temps, la vente paraît préserver l'égalité successorale, puisque son prix, ou ce qu'il en reste après le décès des parents, sera partagé entre les enfants. Désormais, la cohabitation des ménages ne constitue plus la clé de voûte de la transmission et, relativement tôt dans le cycle familial, le successeur devient propriétaire à part entière, il est maitre chez lui et il jouit de l'intimité de son ménage.

Les modalités d'achat varient selon les familles. Toute la question est de savoir si l'on va emprunter ou non, hypothéquer la terre ou non. Louis Michel souligne l'importance probable, dès le XVIII' siècle, de l'endettement foncier des agriculteurs de Varennes et de Verchères ${ }^{16}$. Mais il reconnaît en même temps que l'ampleur du phénomène est sujette à des variations dans le temps, suivant la conjoncture, de même que dans le cycle de la vie d'un individu. Or, justement, dans la génération des agriculteurs nés dans les premières décennies du $\mathrm{XX}^{\mathrm{e}}$ siècle, on se fait un point d'honneur de ne pas emprunter! Toute la fierté de ces agriculteurs se concentre dans cette petite phrase répétée à l'envi: «Je n'ai jamais emprunté!» ou «Mon père n'a jamais emprunté! ${ }^{17}$ » Plusieurs des témoignages recueillis montrent à l'évidence que les plus aisés comme les autres se refusent à emprunter, comme si un même sens de l'honneur, associé peut-être à un même sentiment de précarité, les habitait tous. Le spectre des années de crise, de même que l'influence de l'Église, seraient à l'origine de cette attitude des agriculteurs de la première moitié du $\mathrm{XX}^{\mathrm{c}}$ siècle.

Pour éviter au fils un emprunt qui pourrait rendre précaire son établissement, les parents acceptent souvent un mode de paiement par

15. Sur ce sujet, il faut lire Gérard Bouchard, «La dynamique communautaire et l'évolution des sociétés rurales québécoises aux $\mathrm{XIX}^{\mathrm{e}}$ et $\mathrm{XX}^{\mathrm{e}}$ siècles. Construction d'un modèle», RHAF, 40,1 (été 1986): 51-71. Voir aussi Santerre, loc. cit., 42, note 11, qui remarque à Squatteck la mobilité résidentielle des parents qui vendent leur ferme.

16. Louis Michel, «Endettement et société rurale dans la région de Montréal au XVIII ${ }^{e}$ siècle. Premières approches et éléments de réflexion», dans F. Lebrun et N. Séguin, dir., Sociétés villageoises et rapports villes-campagnes au Québec et dans la France de l'Ouest (XVII-XXe siècles), Actes du Colloque franco-québécois de Québec, 1985 (Trois-Rivières/Rennes, 1987). Santerre remarque la nouvelle pratique de l'hypothèque inversée comme mode de paiement des fermes dans les transmissions, que l'on rencontre aussi dans le comté de Verchères: Renaud Santerre, «De la société rurale à la société urbaine: la sécurité économique des personnes âgées au Québec», Forces, 90 (été 1990): 23-27.

17. Dossiers de famille $\mathrm{n}^{\mathrm{os}} 3,10,12,20,33,34$, etc. 
rente annuelle, payable à la fin des récoltes, à la Toussaint, comme c'est la coutume pour le paiement des dettes. Si toutefois le fils emprunte, le père dispose d'une somme assez rondelette pour lui permettre de faire un placement et d'acheter une maison au village. Les emprunts se généraliseront plus tard avec les politiques des prêts agricoles.

La généralisation de la pratique de la vente des propriétés familiales dans les campagnes québécoises est bien connue des chercheurs. Certains, comme Séguin et Courville, en retracent l'origine dès le $\mathrm{XIX}^{\mathrm{e}}$ siècle et l'associent au recul d'une économie agricole de subsistance et à la commercialisation de l'agriculture. Renaud Santerre, quant à lui, note qu'à Squatteck, la vente a supplanté la donation beaucoup plus tardivement, soit vers 1950, et il associe le recul de la donation à l'arrivée des régimes de retraite ${ }^{18}$. À Verchères, la vente, qui apparaît relativement tôt comparativement à d'autres régions, paraît davantage associée à la commercialisation de l'agriculture, laquelle aurait permis l'augmentation des rentes viagères versées aux parents par leur successeur, afin qu'ils puissent «s'offrir» la possibilité d'un logement séparé.

Il faudrait toutefois nuancer l'importance accordée à la pratique notariale comme révélatrice des mentalités paysannes. Des témoins attachent si peu d'importance à la nature des transactions notariales qui règlent les successions qu'ils les confondent entre elles et, de plus, ils paraissent ignorer la signification de nombreuses clauses ou dispositions. Un témoin, par exemple, montre la donation de son grand-père à son père en affirmant qu'il tient en main l'acte de la vente faite à son père. La donation, en effet, était faite contre rente viagère, équivalente, pour notre témoin, à un prix de vente payable par annuités. Un autre, qui a assisté à l'arrangement de famille en présence du notaire, soutient que son père avait fait un testament, alors qu'il s'agit en réalité d'un acte de vente pour le prix d'un dollar, passé à l'âge de 82 ans, il est vrai.

Une étude de la place du notaire dans les arrangements familiaux, de même qu'une étude de l'évolution de la fiscalité des successions, permettraient de raffiner nos interprétations des changements de la pratique notariale des successions. Dans une certaine mesure, les témoignages oraux suppléent à cette lacune. C'est d'ailleurs sur eux, essentiellement, que repose la suite de cet article, où il sera traité des tensions importantes générées par les nouvelles pratiques dans les relations

18. Serge Courville et Normand Séguin, Le monde rural québécois au XIXe siècle (Ottawa, Société historique du Canada) (47): 23. Renaud Santerre, loc. cit., note 14. 
familiales et des mécanismes d'accommodement mis en œuvre pour ménager une transition entre les anciens et les nouveaux usages.

\section{LONGUE TRANSITION ET MÉCANISMES RÉGULATEURS}

Après la vente du bien, il demeurait difficile pour le père de tout quitter, surtout s'il n'avait pas encore atteint l'âge de se retirer: il perdait trop précocement sa raison de vivre et son statut. De l'avis de plusieurs témoins, quitter sa maison, n'être plus maître chez soi et ne plus travailler sa terre constituaient pour un homme la plus grande des privations.

Le changement des comportements à l'égard de la cohabitation n'est pas allé de soi. Accompli progressivement sur une longue période, il fut à l'origine de bien des drames familiaux, dont voici brièvement quelques exemples. Parlant de son grand-père, un témoin affirme qu'en 1905, ce riche agriculteur, âgé de 75 ans, a opposé à son fils une telle résistance, qu'il s'est laissé mourir plutôt que de céder aux pressions de son fils qui le poussait à partir. «Je ne partirai pas d'ici!», disait-il. Et il est mort effectivement avant la date qui avait été prévue pour le déménagement. Sa veuve a dû partir seule pour le village. Un autre agriculteur s'est suicidé, à l'âge de 54 ans, alors que son successeur prenait femme. Il n'était pas prêt à partir pour le village, déclare sa sœur. Lui-même avait gardé son vieux père jusqu'à ce que celui-ci, âgé de 80 ans, ne se résigne enfin à partir pour le village, après la naissance du quinzième enfant du jeune ménage!

Dans d'autres cas, le drame est plus diffus. Il peut aussi toucher les fils successeurs. Des parents qui ne se résignent pas à partir peuvent condamner leur successeur à un mariage tardif quand ce n'est pas au célibat définitif ${ }^{19}$. «C'était rare les épouses qui voulaient rester avec les parents», soutient un autre témoin, évoquant la période autour de la dernière guerre. Pour ces femmes, la cohabitation n'était pas envisageable, même avec une seule personne de la famille du mari: «Je mariais François, pas sa sœur!» D'après les témoins, la réticence opposée à la cohabitation provenait le plus souvent de la future épouse. Il est vrai que cette "pièce rapportée», comme on dit, intervenait dans un cercle fermé où son arrivée remettait en cause les rôles de chacun, ainsi qu'un mode de relations convenu, construit sur de nombreuses années. Les relations entre belles-mères et belles-filles étaient particulièrement complexes. Selon un témoignage, chaque fois qu'elle devait aider sa belle-fille, une belle-mère, tout en s'exécutant,

19. Les drames familiaux sont tirés des dossiers de famille 31 et 35 . Les cas de célibat ou de mariage tardif des successeurs se présentent dans les dossiers 3,10 , 12, etc. 
disait sèchement: «Servante, faites-vous valoir!» Rentière, elle estimait qu'il n'était pas de sa condition de devoir travailler.

Le changement d'attitudes vis-à-vis de la cohabitation se réalise très lentement. Il prend ses racines dans le $\mathrm{XIX}^{\mathrm{e}}$ siècle et ce n'est qu'après la Deuxième Guerre mondiale que toute forme de cohabitation parut odieuse aux jeunes mariés. Pour les couples d'avant-guerre, disons qu'ils s'en accommodaient lorsqu'il le fallait, avec plus ou moins de bonne grâce, à moins que ce ne soit par la force des choses, comme ce fut le cas dans l'extrême pauvreté et, notamment, dans les années de crise.

Un mécanisme régulateur permettait de retarder de quelques années l'inévitable échéance du départ des parents. Un partage des revenus «à moitié» entre le père et le fils, qui jouissait ainsi d'un minimum d'autonomie financière, rendait viable la période de cohabitation. On disait alors que le fils était le fermier de son père. Mais une telle organisation communautaire du travail et des revenus n'allait pas sans problèmes, particulièrement pour le jeune couple toujours maintenu en état de minorité et dont les revenus devenaient nettement insuffisants au fil des naissances des enfants. De plus, il arrivait que les termes de l'entente verbale ne soient pas respectés. Le père, qui gardait la main haute sur la bourse, devait donner à son fils sa part des revenus des récoltes, à la Toussaint. Cette partie de l'entente était généralement respectée, semble-t-il. Par contre, le partage des revenus d'appoint posait souvent problème. Par exemple, après l'abattage d'un animal, il pouvait arriver que le père retienne pour lui seul le prix de la vente d'un veau ou d'un cochon. C'est ainsi que de grignotage en grignotage de ses revenus, la frustration du jeune ménage allait croissant $^{20}$.

Une autre stratégie d'aménagement de la phase communautaire provenait de l'initiative du fils. Pour se ménager un espace d'autonomie sur la terre de son père, le fils pouvait se lancer dans un petit commerce lucratif, qui exigeait peu en surface de terre, tel un élevage d'abeilles ou de rats musqués. En cas de mésentente grave, le petit commerce d'appoint devenait refuge. «Pour lui, y avait rien que ses abeilles!», dit-on souvent de ceux qui s'y enfermaient jusqu'à ne plus s'intéresser à quelqu'autre travail que ce soit ${ }^{21}$.

Mais rien de tout cela n'eut raison des tensions importantes engendrées par la communauté de vie et de travail entre les deux générations. La cohabitation ne durait qu'un temps. Après deux ou

20. Dossiers de famille $\mathrm{n}^{\mathrm{os}} 10,12,18,20$, etc.

21. Dossiers de famille ${ }^{\text {os }} 10,18$ et 31 . 
trois ans, le fils, mettant les poings sur la table, disait à ses parents: «Ou vous partez, ou je pars!» Assez curieusement, c'est toujours en ces termes que les faits sont racontés. Si les parents persistaient à vouloir rester, c'est le fils qui partait avec sa famille! C'est ainsi que l'on a pu voir, parfois, deux ou trois fils se succéder aux côtés du père. D'après quelques témoins, le partage des revenus était le problème majeur des relations père-fils et il serait à l'origine de nombreux départs de jeunes ménages, après quelques années de cohabitation tendue. Cela dit, dans la majorité des cas, les parents finalement se résignaient, vendaient la propriété à leur fils et partaient avec leurs enfants célibataires. Le successeur était enfin seul maître chez lui.

\section{ÉMERGENCE D'UNE NOUVELLE PHASE DANS LE CYCLE FAMILIAL}

De façon générale, la première génération à partir pour le village vit assez mal ce bouleversement. Pourtant, plusieurs en tireront un capital social non négligeable. Pour ces agriculteurs, pouvoir vivre sans travailler constitue l'une des plus évidentes manifestations de la réussite. Mais encore, faut-il que ça se sache! Or, la vitrine la plus parfaite n'est-elle pas offerte par le village?

Un témoignage exprime bien la complexité des enjeux psychologiques et sociaux en cause. Laissons donc la parole à une célibataire, aujourd'hui à la retraite, fille d'une des familles les plus riches d'un village de l'arrière-pays, que nous appellerons Jeanne. Lorsque son frère qui reprenait la ferme s'est marié, ses parents, sa sœur aînée et elle-même, encore mineure, ont dû quitter la maison ancestrale pour le village.

En 1938, lorsque mon frère s'est marié, cela voulait dire pour nous qu'il fallait partir. Au village, un homme n'a plus rien à faire. Il perd tout son prestige. Il n'y a pas d'avantage au village, sauf pour la femme. Elle peut aller à la messe tous les matins! On a eu l'aqueduc au village en 1990. Avant, on avait un puits artésien, l'eau était sulfureuse. Et puis on était obligé d'acheter le lait! Mes parents n'avaient pas le choix presque. De rester avec le fils marié, ça crée des conflits. Les choses ne se feront pas comme vous avez fait. Si vous gardez la main haute, vous restez là!

La vie au village n'apparaît pas ici sous son meilleur jour. Il faut dire que ce village accusait un certain retard sur les autres agglomérations dans le développement des services. À un autre moment de l'entretien, Jeanne ajoutera que la vieille maison de pierre qu'ils quittaient était froide, délabrée et n'avait pas l'électricité; comme quoi 
les rapports au vieux bien de famille sont plus ambigus qu'on ne le laisse croire.

Passer de la campagne au village, à la retraite, était une manière d'afficher sa réussite sociale. Dans la suite de l'entrevue, Jeanne ajoutera, contrairement à ce qu'elle avait dit plus tôt, qu'il était très bien vu, pour un homme à la retraite, de vivre de ses rentes au village. On considérait cet homme comme un quasi-bourgeois et il pouvait encore gagner en prestige s'il gardait avec lui quelques-unes de ses filles célibataires: «C'était valorisant pour le père de garder ses filles à la maison! Il était capable de nourrir sa famille! Quand il allait fumer sa pipe avec les autres au village, c'était quelqu'un, il était pas tout seul 22 !»

Par ailleurs, le village exerçait plus prosaïquement un pouvoir d'attraction suffisamment fort pour venir à bout de la résistance des parents qui hésitaient à faire le saut du départ. En effet, sauf quelques rares agglomérations moins développées, le village attirait par ses services: l'aqueduc, l'électricité, l'église, la poste et le magasin général. La proximité des services était d'autant plus appréciée que l'entretien des routes, l'hiver, a laissé longtemps à désirer. L'historien géographe Serge Courville raconte si bien la vie des agriculteurs retraités au village qu'il n'apparaît pas utile d'aller plus loin sur ce terrain ${ }^{23}$.

Avec le départ des parents, le cycle de la vie familiale sur la terre est brutalement interrompu. L'on a retiré aux parents toute une tranche de vie sur leur propre bien. C'est désormais le prix à payer pour la continuité de la famille sur l'exploitation. De la scission des familles, résulte une nouvelle structure de ménage reconstruite autour des parents entourés de leurs enfants adultes célibataires. Une figure s'affirme, qui est celle, bien connue, du «bâton de vieillesse». La responsabilité des vieux parents incombe désormais aux célibataires qui consacrent leur vie «à finir les parents», suivant une expression peu répandue, mais signalée quelques fois à l'île d'Orléans, comme aussi dans certaines régions de France.

«On a dit que j'avais sacrifié ma vie, je ne me suis pas sacrifié, c'était un témoignage de reconnaissance.» C'est un homme qui parle, cette fois. Au recensement de 1901, on dénombre autant d'hommes que de femmes célibataires dans le comté de Verchères. L'analyse des ménages comportant des célibataires adultes montre que plus du tiers

22. Dossier de famille $n^{\circ} 31$.

23. Serge Courville, Entre ville et campagne. L'essor du village dans les seigneuries du Bas-Canada (Sainte-Foy, Presses de l'Université Laval, 1990), 143-146. 
de ces ménages de célibataires comptent aussi les vieux parents ou un parent veuf ${ }^{24}$.

Certains de ces célibataires dont la vie a été consacrée à leurs parents seront parfois oubliés dans les dernières volontés des parents. Après la mort des parents, leur situation pourra être désastreuse. Ambiguïté des relations d'interdépendance obligées? Quoi qu'il en soit, la communauté désapprouve toujours une telle attitude. Généralement, les parents léguaient aux célibataires l'usufruit de la maison du village ou, dans certaines circonstances, l'usufruit de la maison de ferme. Ils pouvaient aussi leur léguer l'usufruit de l'argent placé après la vente de la propriété. En somme, l'héritage des célibataires correspondait à celui de la mère veuve. L'usufruit se transmettait de la mère veuve aux célibataires qui restaient avec elle. Au tournant du siècle, on ne donnait jamais un bien en toute propriété aux célibataires, car, ne se reproduisant pas, ils ne transmettront pas. Comme par une sorte de résistance du sentiment lignager, cette pratique coutumière va mettre beaucoup de temps à évoluer vers un mode de transmission indifférent au statut matrimonial des héritiers.

\section{ÉCHEC À LA REPRODUCTION}

Dans certains cas, le refus de quitter le vieux bien patrimonial s'accompagne d'un repli sur la famille d'origine, ce qui aura pour effet de rompre la chaîne de la transmission familiale. $\mathrm{Au} \mathrm{XX}^{\mathrm{e}}$ siècle, une petite partie des vieilles fermes familiales ne seront pas transmises parce que les parents n'ont consenti ni à céder la place ni à intégrer une épouse au sein de la famille. Citons parmi d'autres, l'exemple des Hébert, une vieille famille du pays, très bien vue, assez aisée au début du siècle, mais qui connut par la suite un revers de fortune ${ }^{25}$. Les Hébert accusèrent assez durement les années de crise, au moment même où plusieurs garçons arrivèrent en âge de s'établir. Ils étaient douze enfants, nés entre 1895 et 1915 . Plus de la moitié sont demeurés célibataires. Trois de ces célibataires se sont fixés sur le bien de famille avec les parents: l'aînée des filles, ainsi que le premier et le dernier des garçons, tous deux cultivateurs. Chaque fils et chaque fille qui se mariait devait quitter la maison. Il régnait dans cette maison un climat de méfiance vis-à-vis du monde extérieur et vis-à-vis du

24. En 1901, on compte près de $25 \%$ d'hommes et de femmes célibataires âgés de plus de 35 ans et autant d'hommes que de femmes. Trente-sept pour cent de ces célibataires vivent avec leurs seuls vieux parents, $15 \%$ avec leurs parents et leur frère successeur marié. Source: manuscrit du recensement canadien de 1901, d'après l'analyse de Martine Hamel.

25. Dossier de famille $n^{\circ} 10$. 
mariage. «Il faut croire tout le monde honnête, mais faut se méfier de tout le monde», disait le père. Quant à la mère, elle avait coutume de dire à ses garçons qu'un homme «qui ne se marie pas à sa première folie ne le fera jamais, après, il comprend trop!»

À la fin des années 1930, Paul le fils dernier né, bien qu'il ait fait, à Montréal, des études avancées qui le poussaient hors du nid, est revenu vivre sur le bien familial, alors que sa sœur et son frère aînés s'y trouvaient aussi. Les difficultés familiales et la déclaration de la guerre, sans doute, furent pour quelque chose dans cette décision. Paul comprenait parfaitement le prix de ce retour à la maison natale, qu'il affirme avoir consenti librement, en témoignage de reconnaissance. Il avait pleine conscience que ses parents ne céderaient rien de leur vivant ni ne quitteraient la maison et il comprenait cette attitude. La mère est décédée la première et le père, enfin, a cédé la propriété, quelques mois avant son décès, alors qu'il était âgé de 82 ans et malade depuis de nombreuses années. De son propre aveu, du vivant de ses parents, Paul était considéré et se considérait lui-même comme un mineur.

Bien que les occasions ne lui manquèrent pas, «le Géant blond», comme l'appelaient les filles, ne s'est pas marié. Selon toute apparence, il ne l'a jamais réellement souhaité. Son discours sur les femmes et le mariage est riche d'enseignement. «J'étais réservé. J'ai toujours respecté les femmes. C'est pour ça qu'elles m'aimaient.» Quant au mariage, «c'était une vie de sacrifice! On était unis pour le meilleur et pour le pire [...] C'était toute une responsabilité d'être père de famille: un bon père de famille, qui est catholique, si y est pas sauvé, c'est qu'il n'y a pas de justice!»

Ajoutons que cette famille de célibataires était vue dans le voisinage comme un modèle. "Chez eux, il n'y avait pas de discordance!» La valorisation d'un tel modèle familial tend à accréditer un modèle culturel où la famille est la valeur suprême, comme l'écrit Chantal Collard dans une étude sur le célibat dans la région de Charlevoix.

La famille consanguine est la valeur suprême. L'égalité de tous à l'intérieur de cette cellule est pensée en dépit des différences évidentes de destins entre germains. Cette cellule chaleureuse est sous la houlette ferme des parents. La dynamique familiale encourage le dévouement, l'attachement et l'identification à cette famille d'origine qu'on ne quitte qu'à regret ${ }^{26}$.

26. Chantal Collard, «'Nous on n'a pas d'enfants, on a juste nos ancêtres...' Les célibats laïques et religieux dans le comté de Charlevoix au Québec (1900-1960)», Anthropologie et sociétés, 18,1 (1994): 12. 
Il est difficile, à ce stade de la recherche, d'évaluer la diffusion de ce dernier modèle d'organisation familiale. Mais quelques pistes d'interprétation sont suggérées par plusieurs témoignages concordants, qui voient le célibat contraint de certains enfants des familles nombreuses comme la manifestation d'un abus d'autorité de la part des parents. D'aucuns ajoutent qu'il est plus facile pour les parents de garder la main mise sur la propriété lorsque le successeur est célibataire.

\section{CONCLUSION}

Deux modèles de stratégies familiales coexistent, à la fin du XIX ${ }^{\mathrm{e}}$ siècle, dans le comté de Verchères. Dans le premier modèle, la transmission est opérée très tardivement, après le décès des parents, et dans l'autre, au contraire, le bien est transmis du vivant des parents, vers le mariage du successeur. Dans les deux cas, le successeur est choisi parmi les derniers garçons de la famille et son mariage est une condition sine qua non de la succession. La cohabitation des deux ménages qui se relaient sur le bien est d'usage, tout comme l'organisation communautaire du travail. Seul le chef de famille est en droit propriétaire de l'ensemble du bien, mais, dans les faits, il partage ce droit avec tous ceux qui «vivent en famille» avec lui. Le mode d'organisation familiale crée une sorte de droit d'usage en indivision pour ceux qui restent «en famille», ce droit étant souvent fixé légalement dans le droit d'usufruit en faveur des veuves et des célibataires.

$\mathrm{Au} \mathrm{XX}^{\mathrm{e}}$ siècle, sauf pour ce qui regarde le rang du successeur dans la fratrie, beaucoup de choses vont changer. Toutes les familles n'emboîteront pas le pas de cette petite révolution des usages de la transmission. Des parents qui s'obstinent à rester sur le bien en paieront le prix dans l'échec de la reproduction familiale. Cependant, pour la majorité des familles, il en ira différemment. La nouvelle pratique de la vente de la propriété au fils successeur agit comme un révélateur des nouveaux usages et des nouvelles mentalités. Dans les premières décennies du $\mathrm{XX}^{\circ}$ siècle, l'exclusion quasi obligée des parents opère une rupture dans le cycle familial qui entraîne la faillite d'un mode de solidarité entre les générations où donateurs et donataires étaient engagés dans une relation d'obligations réciproques qui ne s'éteignaient qu'avec la mort des parents. La solidarité intergénérationnelle n'est pas morte pour autant. Elle ressurgit, mais pour se retrouver du côté des exclus, le support des parents incombant désormais aux enfants célibataires.

Il est instructif de comparer les pratiques coutumières de Verchères à d'autres pratiques observées dans des régions situées au nord et au sud de l'ouest de la France. Martine Segalen, qui a travaillé dans un pays de 
fermage de la Bretagne, remarque la même coutume de la transmission à un fils né vers la fin d'une grande fratrie ${ }^{27}$. Elle fait observer qu'un tel usage correspond à un cycle familial long dans lequel les parents restent actifs et conservent longtemps leurs responsabilités et leur contrôle sur la famille. Certes, les parents peuvent jouir longuement d'un contrôle qui s'exerce sur une longue fratrie. Par contre, la transmission en fin de cycle réduit la durée de la phase de cohabitation inhérente au processus de succession. La coïncidence ou la quasi-coïncidence entre la retraite du père et la reprise par le fils contourne le conflit de générations qui risque de surgir lorsque père et fils doivent travailler ensemble le même bien, pour bon nombre d'années.

Ces observations prennent tout leur relief dans une comparaison avec les pays de coutume de transmission à l'aîné, comme les Pyrénées et le Quercy, par exemple. Dans le Quercy, le père cédait une partie de son bien dès le mariage de son fils aîné, ce qui constituait en quelque sorte une garantie de la succession future. Les rapports père-fils étaient alors bien différents, car le père, en pleine possession de ses moyens au moment de la transmission, demeurait actif encore longtemps. On disait, dans ces régions, que l'héritier était l'associé de son père. Ce n'est pas le cas du comté de Verchères, puisque le père cède son bien vers l'âge de la retraite, sinon plus tard. Une succession tardive dans la vie du père peut correspondre à une autonomie relativement précoce pour le fils puisqu'il se retrouve assez rapidement maître du bien et non pas l'associé de son père.

Dans le Québec d'aujourd'hui, où la vente du bien s'est généralisée, il n'y a pas de successeurs-associés, mais des successeurspatrons, si l'on peut dire. Ce modèle séduisant, toujours souhaité par le jeune ménage, n'a toutefois pas toujours été atteint sans tensions ni sans déchirements pour les parents exclus qui ont souvent demandé et obtenu un sursis, dans une phase d'association transitoire.

Pourtant, malgré les différences observées quant au calendrier de la succession, les parents du Québec et ceux du Quercy expriment en des termes très semblables leur anxiété vis-à-vis de l'éventualité de leur démission de biens: «Avant de se coucher pour l'éternité, faut pas se déshabiller trop vite!», soutient un témoin québécois, tandis que, dans le Quercy, on déclare: «Donner et mourir, on a toujours le temps ${ }^{28}$ !»

27. Martine Segalen, «Cycle de la vie familiale et transmission du patrimoine en Bretagne», Ethnologie française, 4,4 (1978): 277.

28. Diane Gervais, Les pratiques coutumières de la transmission patrimoniale, dans un canton du Quercy, de 1750 à nos jours, thèse de doctorat (anthropologie), Université de Montréal, 1990, 285 p. 


\section{ANNEXE \\ Dossiers de familles cités}

Dossier $n^{\circ}$ 3: 184 arpents de terre faite.

Génération 1 (XIX ${ }^{\mathrm{e}}$ siècle) 7 enfants. Transmission au fils plus jeune par donation, un an avant son mariage.

Génération 2 ( $\mathrm{XX}^{\mathrm{e}}$ siècle) 13 enfants. Transmission aux deux derniers fils célibataires, par la réserve d'un droit d'usage sur les terres vendues à une firme de spéculation immobilière. Partage égal du prix de vente.

Dossier $n^{\circ}$ 10: 80 arpents de terre faite.

Génération 1

7 enfants. Transmission au fils plus jeune, marié, par testament.

Génération 2

12 enfants. Transmission à la fille aînée célibataire, au moyen d'une vente pour $1 \$$.

Dossier $\mathrm{n}^{\circ}$ 12: 90 arpents de terre faite.

Génération 1

Succession vacante qui avait été achetée par un émigré à son retour des États-Unis. Sans enfant, il vend à un fils de cultivateur de la paroisse voisine.

Génération 20 enfants. Le père, malade, travaille successivement avec ses deux fils aînés. Chacun est parti après avoir vécu deux ans dans la maison paternelle avec sa jeune épouse. Transmission au fils plus jeune, marié tardivement, par la mère veuve, qui vend à 72 ans, pour le prix de l'hypothèque.

Dossier $\mathrm{n}^{\circ}$ 18: 91 arpents de terre faite.

Génération 1

Génération 2
Bien reçu par la donation d'un couple de cultivateurs sans enfant, faite à un fils de cultivateur sans terre.

10 enfants. Le dernier des garçons, marié dans la maison de ses parents, cultive avec son père un bien qui ne lui sera jamais transmis, mais sera vendu par le père, à la fin de sa vie. 
Dossier $\mathrm{n}^{\circ}$ 20: 120 arpents de terre faite.

Génération 1

Génération 2
Journaliers sans terre avec 4 enfants. Le fils plus jeune achète la terre pour s'y installer avec ses parents et sa famille.

8 enfants, dont 7 garçons. Tous établis sur des terres voisines, «par ajouts», sauf le plus jeune qui succède à son père en achetant la propriété.

Dossier $\mathrm{n}^{0}$ 31: 5 exploitations, dont 4 travaillées par des fermiers.

Génération 1

Génération 2
2 religieuses et un fils. Transmission au fils, par étapes, allant du contrat de mariage au testament.

6 enfants. Transmission par testament entrầnant un partage inégal entre garçons et filles. Vieux bien paternel transmis au fils aîné, seul cultivateur.

Dossier $\mathrm{n}^{0}$ 33: plus ou moins 90 arpents.

Génération 1

Génération 2
9 enfants dont 3 garçons. Le $9^{\mathrm{e}}$ né succède à son père, par achat, après avoir été le fermier de son père.

6 enfants. Pas de transmission. Bien vendu après le décès prématuré du père malade.

Dossier $\mathrm{n}^{\circ}$ 34: 2 terres, dont une de 55 arpents, et deux terres louées, une maison «à deux côtés».

Génération 1

Génération 2

\section{Inconnu.}

Nombre d'enfants inconnu. Un fils achète, en 1921, la terre de son père, puis en 1929 , celle de son beau-père.

Dossier $\mathrm{n}^{\circ}$ 35: 2 terres qui font 150 arpents.

Génération 1

Génération 2

Génération 3
Nombre d'enfants inconnu. Transmission par la vente du père à son fils.

Nombre d'enfants inconnu. Transmission par la vente du père, âgé de 79 ans, à son fils.

15 enfants. En 1952, transmission par la vente à deux fils, en indivision, les $11^{\mathrm{e}}$ et $13^{\mathrm{e}}$ nés. 